\title{
Evolución reciente de los recursos hídricos en la cabecera del río Mero (Galicia, noroeste de España)
}

\author{
Recent evolution of water resources in the headwater \\ of the Mero basin (Galicia, NW Spain)
}

\author{
$\mathrm{M}^{\mathrm{a}}$ LUZ RODRÍGUEZ-BLANCO*1, M $^{\mathrm{a}}$ MERCEDES TABOADA-CASTRO ${ }^{2}, \mathrm{M}^{\mathrm{a}}$ \\ TERESA TABOADA-CASTRO ${ }^{3}$ \\ ${ }^{1}$ Departamento de Historia, Arte y Geografía, Grupo de Estudios de Arqueología, Antigüedade e \\ Territorio GEAAT, Universidad de Vigo, Campus As Lagoas, 36310 Ourense, España \\ ${ }^{2}$ ETSIIAA, Área de Edafología y Química Agrícola, Universidad de Valladolid, 34004 Palencia, España \\ ${ }^{3}$ Facultad de Ciencias, Centro de Investigaciones Científicas Avanzadas (CICA), Universidade da \\ Coruña 15071 A Coruña, España \\ * Autor de contacto: maria.luz.rodriguez.blanco@uvigo.es
}

https://doi.org/10.17979/cadlaxe.2020.42.0.7309

Recibido: 13/11/2020, Aceptado: 11/12/2020

\begin{abstract}
The trends analysis of hydroclimatic variables (rainfall, temperature, streamflow) in headwater catchments provides basic scientific criteria necessary for water resource planning and management. However, relatively few studies have been carried out in these relatively nearnatural systems, i.e. with little or no alteration by humans. In this context, the overall aim of the present study is to characterize the recent trend of stream discharge in a headwater catchment of the Mero Basin (NW Spain). The results showed no significant decrease in annual stream discharge. However, a significant decrease in summer and autumn stream dis-
\end{abstract}


charge and an increase in the duration and severity of low flow was detected. In this study, a parallelism between hydrological processes and rainfall was not appreciated. So, the trends observed for streamflow are probably related to upward trend in temperature, by enhancing evapotranspiration.

Key words: hydrological resources, evolution, trends, headwater catchment, NW Spain

\section{RESUMEN}

El estudio de la evolución y tendencia de variables hidro-climáticas en cuencas de cabecera proporciona criterios científicos de base necesarios para la planificación y gestión sostenible de los recursos hídricos. Sin embargo, se han realizado pocos estudios en naturales sistemas, es decir cuencas donde la actividad humana no repercute notablemente en el funcionamiento hidrológico natural. En este contexto, el objetivo del presente estudio es caracterizar la tendencia reciente del caudal en una cuenca de cabecera del Mero (noroeste de España). Los resultados no permiten confirmar la existencia de una tendencia regresiva del caudal a escala anual. Sin embargo, se detecta una disminución significativa en el caudal en verano y otoño, así como un incremento en la duración y severidad de los caudales bajos. En este estudio no se aprecia un paralelismo entre los procesos hidrológicos y las precipitaciones, por lo que el descenso del caudal y el incremento de las frecuencias de días con baja descarga podría estar relacionado con el incremento térmico observado, al intensificar procesos como la evapotranspiración.

Palabras clave: recursos hídricos, evolución, tendencias, cuenca de cabecera, noroeste de España 


\section{INTRODUCCIÓN}

Los recursos hídricos y su disponibilidad son el eje central del desarrollo de las sociedades y de los ecosistemas, pues el agua constituye un elemento esencial para la vida. De ahí que la propia Directiva Marco del Agua (Directiva 2000/60/CE) señale que el agua no es una bien comercial como los demás, sino un patrimonio que hay que proteger, defender y tratar como tal. La sociedad actual también demanda cada día más un desarrollo sostenible y respetuoso con el medio ambiente, siendo primordial en materia de agua conocer los recursos disponibles.

La preocupación actual existente sobre la disponibilidad de agua es evidente sobre todo en el ámbito científico. Prueba de ello, es el amplio número de trabajos realizados en todo el mundo para analizar la evolución y variabilidad de las series de caudales (MACHÍN ET AL., 2005; VILLARINI et al., 2011; BERTOLA et al., 2020), lo que constituye una tarea fundamental en la evaluación de los recursos hídricos.

Las cuencas de cabecera son de extrema importancia para los ambientes fluviales pues encierran cursos de agua en el nacimiento o zona de origen de un río y cualquier alteración dentro de su territorio modifica el funcionamiento hidrológico de la cuenca. Además, pueden servir como sectores de control de cambios a las variaciones climáticas, debido a la menor confluencia de factores antrópicos. Por todo ello, el estudio de su evolución y de las tendencias de las variables involucradas en la generación de escorrentía en estos sistemas son fundamentales para la planificación y gestión sostenible de los recursos hídricos (MACHÍN et al., 2005; SVENSSON et al., 2005). Sin em- bargo, los estudios en cuencas naturales son más escasos a nivel mundial (HANNFORD et al., 2008; STAHL et al., 2010; WILSON et al.; 2010; MARTÍNEZ-FERNÁNDEZ et al., 2013), debido a la dificultad de encontrar áreas poco afectadas por alteraciones antrópicas. Esta escasez de conocimientos se traslada también a la Península Ibérica, y Galicia no es ajena a ello; pues la mayoría de los ríos carecen de registros de caudal lo suficientemente largos como para llevar a cabo el análisis de régimen fluvial.

El principal objetivo de este trabajo es analizar la evolución reciente de los caudales en una subcuenca del tramo alto del río Mero, el tercer río más largo de Galicia (noroeste de España). Para ello, se examina a distintas escalas temporales i) la variabilidad temporal de las principales variables climáticas, precipitación y temperatura, por su indudable relación con los aportes fluviales, ii) las tendencias experimentadas por los caudales, prestando especial atención a los caudales altos y bajos, y iii) la relación que guarda la evolución de los recursos hídricos con las variables climáticas. La elección del área de estudio está fundamentada en: i) su carácter de cuenca poco modificada por el hombre, en cuanto a la ausencia de embalses, presas, etc., y por tanto, con una serie de caudal representativos del régimen natural o próximo al natural, ii) sus características que responden al arquetipo del entorno rural gallego: pequeños núcleos de población con viviendas unifamiliares muy dispersas, y iii) el interés que presenta la cuenca desde el punto de vista científico, ya que se encuentra aguas arribas del embalse de Cecebre, a partir del cual se abastece de agua potable a la ciudad de A Coruña y parte de su área metropolitana. Dicho embalse, además, tiene un alto valor como ecosistema 
y se encuentra entre los lugares incluidos en la Red Natura 2000 de la Unión Europea.

\section{MATERIAL Y MÉTODOS}

\section{Área de estudio}

La cuenca del río Corbeira $(16 \mathrm{~km} 2)$ se sitúa en la cabecera del río Mero, aproximadamente a $30 \mathrm{~km}$ de la ciudad de A Coruña, en la confluencia de los términos municipales de Oza-Cesuras y Abegondo, a una latitud de $43^{\circ} 13 ` 2,3^{\prime \prime} \mathrm{N}$ y longitud de $8^{\circ} 13 ` 43,9$ ” O (Figura 1). Se origina a 470 $\mathrm{m}$ sobre el nivel del mar en el término de Santa María de Dordaño, recorriendo 10,3 $\mathrm{km}$ hasta el punto de su desembocadura en la confluencia con el río Mero en el lugar de Cullergondo (60 m), en el municipio de Abegondo. El relieve es accidentado, siendo la pendiente media de la cuenca del 19\%, mientras que la pendiente media del cauce es del 3\%. Desde el punto de vista geológico, se sitúa sobre un substrato uniforme constituido por materiales pertenecientes al Complejo de Órdenes concretamente esquistos básicos (IGME, 1981), siendo filitas y esquistos biotíticos los tipos petrológicos más comunes del área. Los suelos predominantes son los Umbrisoles y Cambisoles (IUSS, 2015) y se caracterizan por su profundidad y buen desarrollo, una textura limosa o franco-limosa y elevada capacidad de campo. La cuenca del Corbeira es una cuenca rural, caracterizada por la presencia de pequeños núcleos de población dispersa (densidad de población: $35 \mathrm{hab} \mathrm{km} 2$ ), explotaciones agropecuarias escasas y actividad agrícola no intensiva. La superficie dedicada a uso forestal conforma el $65 \%$ de la cuenca, en tanto que el 30\% de la superficie corresponde a superficie agraria útil y está ocupada por praderas y parcelas de cultivo dispuestas en mosaico (Figura 1).

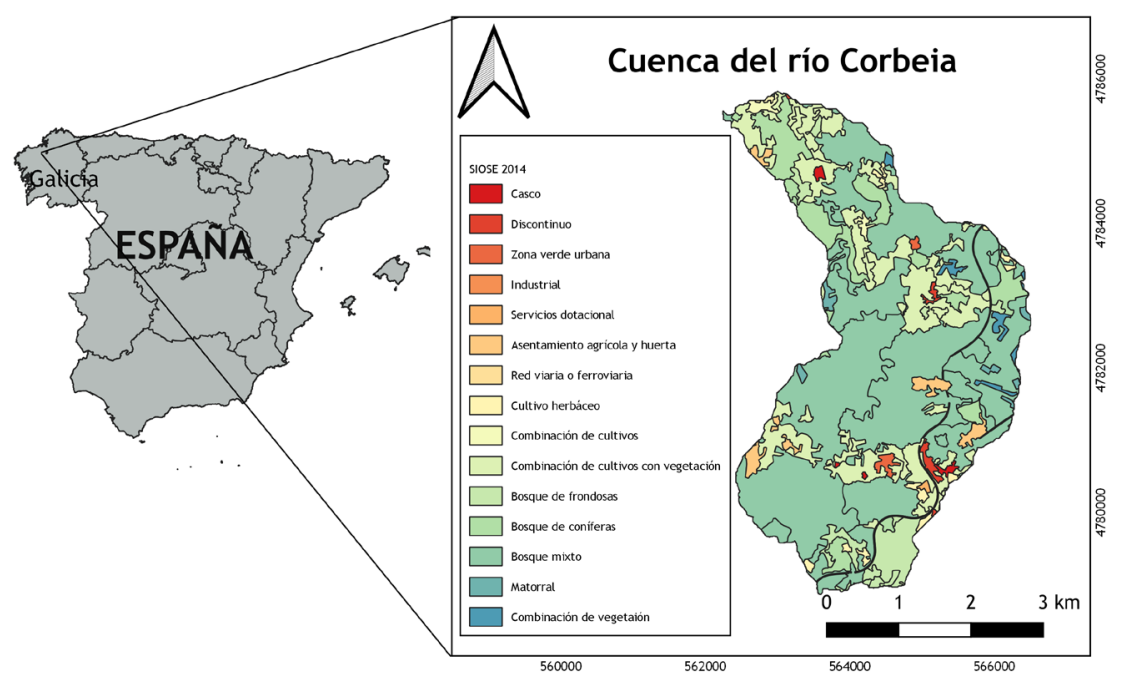

Figura 1. Situación geográfica del área de estudio y distribución de los usos del suelo en la cuenca del río Corbeira. 
El clima de la zona de estudio es Atlántico (oceánico húmedo) con una temperatura media anual de $13{ }^{\circ} \mathrm{C}$ y una precipitación media anual de $1074 \mathrm{~mm}$ (serie histórica: 1983-2019). La distribución estacional de la precipitación es otoño $>$ invierno $>$ primavera $>$ verano, registrándose, en promedio, más $65 \%$ de la precipitación anual entre octubre y marzo.

\section{Metodología}

Para la realización de este trabajo se han utilizado datos climáticos (precipitación y temperatura) e hidrológicos. Las series de datos climáticos fueron tomados de la estación meteorológica de Mabegondo, que se halla instalada cerca del cierre de la cuenca y dispone de un amplio registro de precipitaciones y temperaturas, mientras que los datos hidrológicos (caudal) fueron obtenidos a la salida de la cuenca del río Corbeira entre octubre de 2004 y septiembre de 2019 (15 años hidrológicos). En el cierre de la cuenca se dispone de una estación de aforo con sección fluvial natural, dotada con un sensor de presión modelo ISCO 720 acoplado a un muestreador automático ISCO 6712. El sensor realiza una medida por minuto y a partir de estos datos minutales, el sistema calcula una media aritmética a intervalos de 10 minutos, siendo estos valores medios los que finalmente van a integrar los registros. Los datos diezminutales de nivel se transformaron en caudal a partir de una curva de gastos (relación nivel-caudal) lo que permite disponer de datos de caudal a intervalos de diez minutos.

A partir de los datos diarios de caudal se obtuvieron los caudales bajos y altos en base a los percentiles 0,1 y 0,2 para caudales bajos (OUARDA et al., 2008) y a los percen- tiles 0,8 y 0,9 para caudales elevados (WILSON et al., 2010). Además del caudal medio (Qm) se calcularon los siguientes parámetros para su posterior análisis (Figura 2):

Para caudales bajos:

- Q10 (percentil 0,1): caudal medio diario que es excedido el $90 \%$ del periodo de estudio.

- Q20 (percentil 0,2): caudal medio diario que es excedido el $80 \%$ del periodo de estudio.

- Duración: periodo de tiempo (días) con valores de caudal por debajo de percentil 0,1 (Q10) y 0,2 (Q20).

- Severidad: déficit de caudal, es decir el volumen por debajo de Q10 y Q20 en el periodo de estudio.

Para caudales altos:

- Q80 (percentil 0,8): caudal medio diario que es excedido el $20 \%$ del periodo de estudio

- Q90 (percentil 0,9): caudal medio diario que es excedido el $20 \%$ del periodo de estudio

- Duración (D): periodo de tiempo (días) con valores caudal por encima del percentil $0,8(\mathrm{Q} 80)$ y 0,9 (Q90).

\section{Análisis de tendencias}

El análisis de las posibles tendencias se ha realizado sobre las series hidrológicas y climáticas. Tradicionalmente a la hora de valorar la pendiente de tendencia en series temporales de larga duración ( $>40$ años) se ha hecho uso de técnicas de regresión. En nuestro caso, la ausencia de series de esa magnitud temporal lleva a usar técnicas de extracción de tendencias y validación alter- 
nativas para obtener resultados confiables. En este sentido, la prueba de Mann-Kendall constituye una adecuada opción que se ha utilizado en multitud de estudios debido a su poder de extracción de tendencias confiables sin necesidad de un alto número de muestras (KUNDZEWICZ et al., 2005). Por esta razón, para la detección de las tendencias se ha utilizado la prueba no paramétrica de Mann-Kendall y el método Sen, haciendo uso de la macro "Makensens" desarrollada por la Oficina Meteorológica de Finlandia (SALMI et al., 2002). La presencia de una tendencia ascendente (positiva) o descendente (negativa) y su grado de significación se probó con la prueba de Mann-Kendall y la pendiente de la tendencia (es decir, la magnitud) se estimó con el método Sen (GILBERT et al., 1987). Las tendencias se han analizado a diferentes escalas temporales: anual, estacional y mensual, las dos últimas con el fin de comprobar cuáles son los periodos del año más sensibles. Para analizar la tendencia estacional, el año se ha dividido en cuatro estaciones que comprenden periodos de tres meses completos, así el otoño se refiere a los meses de octubre, noviembre y diciembre), el invierno a los de enero, febrero y marzo, la primavera a los de abril, mayo y junio y el verano a los de julio, agosto y septiembre.

\section{RESULTADOS}

\section{Caracterización hidrológica del periodo de estudio}

El caudal medio del río Corbeira para el periodo estudiado es de $0,18 \mathrm{~m} 3-1$. La variabilidad del caudal es alta, registrándose valores que comprenden desde los $1,41 \mathrm{~m} 3 \mathrm{~s}$ -1 a valores ciertamente bajos $(0,001 \mathrm{~m} 3 \mathrm{~s}$ -1) (Figura 2). Asimismo, la variación de los caudales máximos y máximos instantáneos también es amplia, alcanzándose máximos extremos de 2,12 m3s -1. En lo que respecta a la magnitud y frecuencia de las avenidas, evaluadas a partir de los episodios que exceden determinados umbrales, se advierte que 241 días exceden el coeficiente Qx3 (valor alcanzado cuando el río registra el triple de su caudal medio), lo que señala que solo un 4\% de los datos de caudal son aguas altas. Los eventos que superan por cinco el caudal medio del río representan tan solo el $1 \%$ de los episodios. El caudal máximo diario alcanzado en la serie $(1,41 \mathrm{~m} 3 \mathrm{~s}-1)$ sería equivalente a una crecida de orden 8 . En lo que se refiere a las aguas bajas, es decir periodos de tiempo en los que el caudal del río se encuentra por debajo de los umbrales de Qm/3 (0,060 m3s - $)$, Qm/5 (0,036 m3s - $)$ y $\mathrm{Qm} / 10(0,018 \mathrm{~m} 3 \mathrm{~s}-1)$ se constata que el $25 \%$ de los caudales diarios del periodo estudiado corresponden a aguas bajas. 


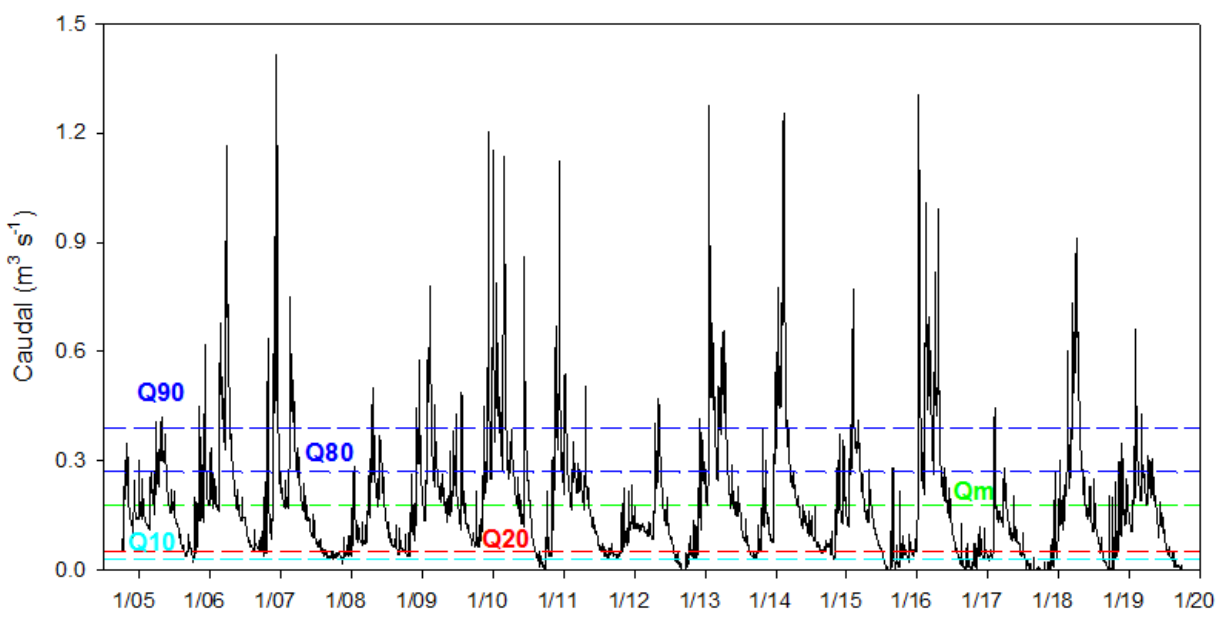

Figura 2. Hidrograma del río Corbeira durante el periodo de estudio y percentiles asociados (Q10, Q20, Q80, Q90). Qm: caudal medio.

La distribución del caudal a lo largo del año corresponde, como cabe esperar, a un típico río de régimen pluvial oceánico (Figura 3), esto es: un periodo de aguas altas en los meses de invierno e inicios de primavera, fruto de la acumulación de las precipitaciones de otoño (que saturan la capacidad de almacenamiento de agua del suelo) y de invierno, y un acusado descenso durante la primavera, hasta alcanzar en verano los meses de aguas bajas. Así, desde junio hasta octubre, el río mantiene valores muy inferiores al caudal medio, debido a la reducción de las precipitaciones durante la estación estival, observándose el mínimo anual en septiembre. Por otro lado, se aprecian altos signos de variabilidad inter-anual del caudal, especialmente en los meses de invierno, cuando el caudal medio es más elevado. El rango de variabilidad es más acusado en los valores por debajo de la media que por encima (Figura 3), lo que señala que los años de mayor caudal condicionan de manera significativa el valor medio de una serie de datos. 


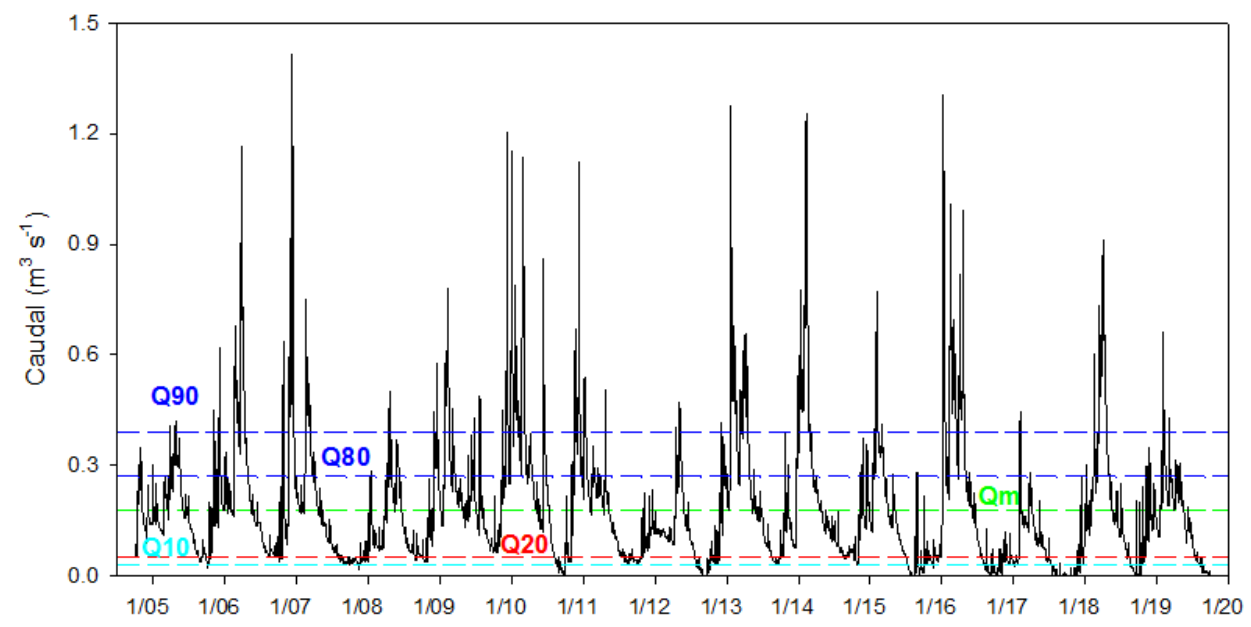

Figura 3. Régimen fluvial del río Corbeira durante el periodo de estudio.

Tendencias en las series de temperatura, precipitación y caudal

La tabla 1 recoge los resultados de los análisis de tendencias de las series de temperatura, precipitación y caudal a escala anual, estación y mensual. Los datos reflejan que la temperatura media anual ha experimentado una tendencia creciente durante el periodo de estudio. De hecho, ha registrado (la temperatura) tendencias positivas en la mayor parte de los meses del año, siendo el incremento más acentuado en los meses de enero, abril, mayo, junio, julio, agosto, seguidos en menor medida, de octubre y marzo. Por ende, primavera y verano han sido las estaciones con un ascenso térmico más pronunciado, mientras que en el invierno el incremento ha sido más atenuado. En otoño se registra un incremento más suave, careciendo este de significación estadística. Las precipitaciones muestran una leve tendencia ascendente en los valores anuales, así como en invierno y en los meses de febrero, marzo, agosto y noviembre. Por el contrario, se observa un ligero descenso en los meses de septiembre y diciembre, aunque los valores de tendencia no han alcanzado el umbral de significación estadística, lo que no permite hablar de tendencias contrastadas. La ausencia de una tendencia clara en las precipitaciones puede deberse a la acusada variabilidad temporal detectada en la evolución de las mismas (coeficiente de variación anual del $21 \%$ ). 


\begin{tabular}{|l|l|l|l|l|l|l|}
\hline \multirow{2}{*}{} & \multicolumn{2}{|c|}{ Temperatura } & \multicolumn{2}{c|}{ Precipitación } & \multicolumn{2}{c|}{ Caudales } \\
\cline { 2 - 7 } & $\begin{array}{l}\text { Kest Mann- } \\
\text { Kendall }\end{array}$ & $\begin{array}{l}\text { pendiente } \\
\text { de Sen }\end{array}$ & $\begin{array}{l}\text { test Mann- } \\
\text { Kendall }\end{array}$ & $\begin{array}{l}\text { pendiente } \\
\text { de Sen }\end{array}$ & $\begin{array}{l}\text { test Mann- } \\
\text { Kendall }\end{array}$ & $\begin{array}{l}\text { pendiente } \\
\text { de Sen }\end{array}$ \\
\hline Anual & $3.69\left(^{* *}\right)$ & 0.037 & 1.48 & 5.242 & -1.09 & -0.004 \\
\hline Otoño & 1.35 & 0.027 & 0.61 & 1.989 & $-2.28\left(^{*}\right)$ & -0.011 \\
\hline Invierno & $1.79(+)$ & 0.027 & 1.43 & 3.688 & 0.20 & 0.002 \\
\hline Primavera & $3.81(* *)$ & 0.055 & -0.15 & -0.198 & -0.49 & -0.003 \\
\hline Verano & $2.60(* *)$ & 0.036 & -0.25 & -0.208 & $-2.67(* *)$ & -0.003 \\
\hline Enero & $2.10\left(^{*}\right)$ & 0.048 & 0.67 & 0.884 & 0.00 & -0.008 \\
\hline Febrero & 0.11 & 0.003 & 1.38 & 1.453 & 0.79 & -0009 \\
\hline Marzo & $1.96(+)$ & 0.027 & 1.35 & 1.157 & 0.20 & -0.020 \\
\hline Abril & $2.33(*)$ & 0.061 & 0.01 & 0.017 & -0.49 & -0.001 \\
\hline Mayo & $2.16(*)$ & 0.048 & -0.04 & -0.042 & -1.09 & 0.011 \\
\hline Junio & $3.29\left(^{* *}\right)$ & 0.068 & 0.48 & 0.257 & -1.29 & 0.003 \\
\hline Julio & $2.06(*)$ & 0.040 & 0.71 & 0.164 & -1.39 & -0.005 \\
\hline Agosto & $2.13(*)$ & 0.033 & 1.20 & 0.372 & $-2.28(*)$ & -0.005 \\
\hline $\begin{array}{l}\text { Septiem- } \\
\text { bre }\end{array}$ & 1.05 & 0.029 & -1.27 & -0.902 & $-2.67(* *)$ & -0.005 \\
\hline Octubre & $1.87(+)$ & -0.017 & 0.69 & 0.873 & $-2.57(*)$ & -0.004 \\
\hline $\begin{array}{l}\text { Noviem- } \\
\text { bre }\end{array}$ & 0.31 & 0.009 & 1.40 & 2.219 & -1.19 & -0.003 \\
\hline Diciembre & 0.28 & 0.007 & -1.08 & -0.980 & $1.68(+)$ & -0.004 \\
\hline
\end{tabular}

Nivel de significación: ***: 0,001, **: 0,01, *: 0,0,5, +: 0,1

Tabla 1. Tendencias en la evolución de las temperaturas, precipitaciones y caudales durante el periodo de estudio.

En lo que respecta al caudal, los resultados muestran una ligera tendencia anual descendente, aunque sin ser estadísticamente significativa (Tabla 1). No obstante, al analizar el comportamiento intra-anual se detectan varias tendencias significativas. En otoño se registra una tendencia regresiva significativa, muy clara en octubre, más débil y carente de significación en noviembre y ligeramente positiva en diciembre. El verano también presenta un retroceso significativo de los caudales, particularmente en lo meses de septiembre y agosto, pues en julio no se alcanza el umbral de significación estadística. Finalmente, el invierno y primavera carecen tendencias estadísticamente significativas (Tabla 1).

\section{Tendencia de los caudales altos y bajos}

En la tabla 2 se muestran los resultados del análisis de tendencias para los índices anuales y estacionales que caracterizan los caudales bajos (Q10 y Q20) y altos (Q80 y Q90). En lo que a los caudales bajos respecta, se aprecia una tendencia positiva signifi- 
cativa en la serie anual, lo que indica que la duración y severidad del periodo de aguas bajas está aumentando en el tiempo. En el caso del análisis estacional, el número de días con caudales bajos ha aumentado significativamente en otoño y verano, mientras que en invierno y primavera ha disminuido, aunque en este caso carece de significación estadística. A diferencia de lo que ocurre con los caudales bajos, el número de días con caudales altos muestra una tendencia decreciente a escala anual, aunque, en este caso, no alcanza el umbral de significación estadística (Tabla 2). De hecho, solo se constata un retroceso significativo de los caudales altos en otoño.

\begin{tabular}{|c|c|c|c|}
\hline & & test Mann-Kendall & pendiente de Sen \\
\hline \multirow[t]{6}{*}{ Anual } & Q10 & $2.79(* *)$ & 6.875 \\
\hline & Q20 & $3.07(* *)$ & 8.429 \\
\hline & Severidad (Q10) & $2.90(* *)$ & 9.379 \\
\hline & Severidad (Q20) & $1.48(* *)$ & 0.011 \\
\hline & Q80 & -0.84 & -1.000 \\
\hline & Q90 & -0.10 & -0.333 \\
\hline \multirow[t]{4}{*}{ Otoño } & Q10 & $2.59(* *)$ & 1.00 \\
\hline & Q20 & $2.38(*)$ & 3.00 \\
\hline & Q80 & -1.50 & -1.444 \\
\hline & Q90 & $-1.89(+)$ & -0.250 \\
\hline \multirow[t]{4}{*}{ Invierno } & Q10 & 1.04 & 0.000 \\
\hline & Q20 & 0.17 & 0.000 \\
\hline & Q80 & 0.25 & 1.000 \\
\hline & Q90 & 0.40 & 0.222 \\
\hline \multirow[t]{3}{*}{ Primavera } & Q20 & 1.04 & 0.000 \\
\hline & Q80 & -1.19 & -0.667 \\
\hline & Q90 & -0.25 & 0.000 \\
\hline \multirow[t]{2}{*}{ Verano } & Q10 & $2.24(*)$ & 4.200 \\
\hline & Q20 & $2.31(*)$ & 4.200 \\
\hline
\end{tabular}

Nivel de significación: ***: 0,001, **: 0,01, *: 0,0,5, +: 0,1

Tabla 2. Tendencias en la evolución de caudales altos y bajos.

\section{DISCUSIÓN}

Los resultados del análisis de tendencias de las variables climáticas apuntan a un ascenso térmico tanto a escala anual como estacional, como ha sido señalado en otros es- tudios en la Península Ibérica (DEL RÍO et al., 2011, MORÁN-TEJEDA et al., 2014). Por el contrario, no se aprecia una tendencia manifiesta para las precipitaciones durante el periodo de observación. En cierto modo, este resultado entraría en contradicción 
con los pronósticos sobre la evolución de la precipitación en el sur Europa, que auguran un descenso de las precipitaciones. No obstante, los resultados están en línea con trabajos realizados en la comunidad gallega. Por ejemplo, RODRIGO y TRIGO (2011) para la estación meteorológica de A Coruña observan una tendencia positiva, aunque no estadísticamente significativa, en la precipitación anual y en otoño y un descenso en la precipitación de primavera y verano. LAGO et al. (2006) utilizando estaciones pluviométricas distribuidas por Galicia, no encuentran una tendencia significativa generalizada en la evolución de las precipitaciones anuales, aunque constatan un cambio en la distribución de las precipitaciones a lo largo del año caracterizada por otoños más húmedos, inviernos menos húmedos y primaveras y veranos más secos.

El análisis de la evolución y tendencia del caudal revela un ligero retroceso de este en la cuenca del Corbeira, aunque sin ser estadísticamente significativo. Estos resultados contrastan con el descenso generalizado de los recursos hídricos reportado para ríos con regímenes de caudal seminatural de la Península Ibérica y del sur de Europa (en el que se incluyen varias subcuencas del norte de España) Por ejemplo, LORENZOLACRUZ et al. (2012), al estudiar una larga base de datos de ríos de montaña españoles no afectados por actividades humanas, detectan una reducción generalizada en el caudal de los arroyos. MARTÍNEZ-FERNÁNDEZ (2013) encuentran una situación similar en un análisis de 74 ríos seminaturales en España, mientras que ZABALETA et al. (2013) destacan tendencias negativas dominantes en varias cuencas del País Vasco. Sin embargo, HAWTREE et al. (2015) en la cuenca de Águeda (región centro-norte de Portugal), con características bastante similares a las del área de estudio, atribuyen la ausencia de un retroceso significativo de los caudales a la presencia de tendencias climáticas compensatorias durante el periodo de estudio.

En este estudio, si bien no se detecta una tendencia a escala anual, si se observa una evolución regresiva del caudal en otoño y verano. Este declive en el caudal medio lleva implícito un notable aumento en la frecuencia y en la severidad (déficit volumétrico) de los caudales bajos, a la par que desciende la frecuencia de días con caudales altos. Es evidente que las precipitaciones ejercen un acusado control sobre el caudal del río (PALLEIRO et al., 2014; RODRÍGUEZBLANCO et al., 2019) y presumiblemente, el pronunciado descenso de los aportes fluviales en otoño y verano debería estar relacionada con cambios en el volumen y patrón de las precipitaciones. Sin embargo, los resultados obtenidos para las precipitaciones no permiten hablar de tendencias contrastadas, por lo que el descenso del caudal y el incremento de las frecuencias de días con baja descarga podría estar relacionado con el incremento térmico observado, que contribuye a mayores tasas de evapotranspiración. Estos procesos a menudo provocan una disminución de los caudales en las cuencas hidrográficas (MARTÍNEZFERNÁNDEZ et al., 2013), en las que también juegan un papel muy importante el crecimiento de la cubierta vegetal (LORENZO-LACRUZ et al.,2012; MEDIERO et al., 2014). De hecho, distintos trabajos llevados a cabo en la Península Ibérica atribuyen parte del descenso en los caudales al aumento en la cubierta vegetal en las cabeceras fluviales (LORENZO-LACRUZ et al.,2012; BUENDIA et al., 2016). 
Las tendencias observadas en la cuenca del río Corbeira pueden ser un reflejo de lo que sucede en la cuenca Mero, lo que sin duda tiene importantes implicaciones para la gestión del agua en la zona de estudio. El embalse Abegondo-Cecebre, que se alimenta principalmente del agua de la cuenca del río Mero, se construyó en la década de 1970 con la finalidad de garantizar la disponibilidad de agua a la ciudad de A Coruña y su área metropolitana durante los períodos de sequía, así como para reducir los riesgos de inundaciones. En los últimos años, se ha producido un aumento sustancial en la población de la zona (de 250,000 a 450,000) lo que supone un incremento de la demanda de agua. Por otra parte, el turismo también está creciendo en la ciudad de A Coruña y sus alrededores, especialmente en verano- cuando la población incluso puede duplicarse, lo que requiere satisfacer una demanda adicional de agua en la época de mayor escasez. De hecho, en periodos particularmente secos el reducido volumen de agua almacenada en el embalse ocasiona restricciones en el uso de agua para determinados fines. Todo ello resalta la necesidad de establecer apropiadas estrategias para la gestión sostenible del recurso agua con el fin de satisfacer y conciliar las necesidades de agua en las actividades humanas con el funcionamiento de los sistemas acuáticos, pues no debemos olvidarnos que embalse tiene un alto valor como ecosistema y se encuentra entre los lugares incluidos en la Red Natura 2000 de la Unión Europea.

\section{CONCLUSIONES}

En este estudio se muestra la evolución reciente del caudal en una cuenca de cabecera del río Mero, un sistema seminatural representativo del clima y las características de uso del suelo en el noroeste de la Península Ibérica. Los resultados obtenidos no permiten probar la existencia de una tendencia manifiesta del caudal a escala anual. No obstante, se constata un retroceso significativo del caudal en otoño y verano, observándose un aumento en la duración y severidad de los caudales bajos en primavera y verano. De la misma manera, se aprecia un descenso de la frecuencia de los caudales altos en otoño. Las tendencias registradas por el caudal no se corresponden con las observadas para las precipitaciones, por lo que el retroceso de los caudales se podría asociar con el aumento térmico, al potenciar procesos como la evapotranspiración.

\section{BIBLIOGRAFÍA}

Bertola, M., Viglione, A., Lun, D., Hall, J., Blösch, G., 2020. Flood trends in Europe: are changes in small and big floods different? Hydrology Earth System Science 24, 1805-1822. https://doi. org/10.5194/hess-24-1805-2020

Buendia, C., Batalla, R.J., Sabater, S., Palau, A., Marcé, R., 2016. Runoff trends driven by climate and afforestation in a Pyrenean basin. Land Degradation and Development 27, 823-838. https://doi. org/10.1002/ldr.2384

Del Río, S., Herrero, L., Pinto-Gomes, C., Penas, A., 2011. Spatial analysis of mean temperature trends in Spain over the period 1961-2006. Global and Planetary Change 78, 65-75. https://doi. org/10.1016/j.gloplacha.2011.05.012

Directiva 2000/60/CE del Consejo, de 23 de octubre del 2000. Marco comunitario de actuación en el ámbito de la política de aguas. Diario Oficial de las Comunidades Europeas, L327/1-72. 
Gilbert, R.O., 1987. Statistical methods for environmental pollution monitoring. Van Nostrand Reinhold, New York.

Hannaford, J., Marsh, T.J., 2008. High-flow and flood trends in a network of undisturbed catchments in the UK. International Journal Climatology 28, 13251338. https://doi.org/10.1002/joc.1643

Hawtree, D., Nunes, J.P., Keizer, J.J., Jaciento, R., Santo, J., Rial-Rivas, M.E., Boulet, A.K., Tavares-Wahren, F., Feger, K.H., 2015. Time series analysis of the long-term hydrologic impacts of afforestation in the Águeda watershed of northcentral Portugal. Hydrological Earth System Science 19, 3033-3045. https:// doi.org/10.5194/hess-19-3033-201

IGME (Instituto Tecnologico Geominero de Espana), 1981. Mapa Geológico de España, 1:50,000. Hoja 45. Betanzos. Spain.

IUSS Working Group WRB, 2015. World Reference Base for Soil Resources 2014, update 2015 International soil classification system for naming soils and creating legends for soil maps. World Soil Resources Reports No. 106. FAO, Rome.

Kundzewicz, Z.W., Graczyk. D., Maurer, T., Pińskwar, I., Radziejewski, M., Svensson, C., Szwed, M., 2005. Trend detection in river flow series: Annual maximum flow. Hydrological Science Journal 50, 797-810. https://doi.org/10.1623/ hysj.2005.50.5.797

Lago, A., Lage, A., Cruz, R., Pérez-Muñurizi, V., 2006. Estudo das variacións de temperatura e precipitación en Galicia nos últimos trinta anos no contexto do cambio climático global. Revista Real Academia Galega das Ciencias XXV, 27-57.

Lorenzo-Lacruz, J., Vicente-Serrano, S.M., López-Moreno, J.I., Morán-Tejeda, E.,
Zabalza, J., 2012. Recent trends in Iberian streamflows (1945-2005). Journal of Hydrology 414-415, 463-475. https:// doi.org/10.1016/j.jhydrol.2011.11.023

Machín, M., Navas, A., Domenech, S., López-Vicente, M., 2005. El río Arlanza en cabecera: Evolución reciente y tendencias en condiciones de cambio global. Cuadernos de Investigación Geográfica 31, 77-95. https://doi.org/10.18172/ cig. 1175

Martínez-Fernández, J., Sánchez, N., Herrero-Jiménez, C.M., 2013. Recent trends in rivers near-natural flow regime: The case of the river headwaters in Spain. Progress Physical Geography 37, 685-700. https:// doi.org/10.5194/hess-14-2367-2010

Mediero, L., Santillán, D., Garrote, L., Granados, A. 2014. Detection and attribution of trends in magnitude, frequency and timing of floods in Spain. Journal of Hydrology 517, 1072-1088. https:// doi.org/10.1016/j.jhydrol.2014.06.040

Morán-Tejeda, E., Lorenzo-Lacruz, J., López-Moreno, J.I., Rahman, K., Beniston, M., 2014. Streamflow timing of mountain rivers in Spain: recent changes and future projections. Journal of Hydrology 517, 1114-1127. https://doi. org/10.1016/j.jhydrol.2014.06.053

Ouarda, T.B.M.J., Charron, C., St-Hilaire, A., 2008. Statistical models and the estimation of low flows. Cannadian Water Resources Journal 33, 195-206. https:// doi.org/10.4296/cwrj3302195

Palleiro, L., Rodríguez-Blanco, M.L., Taboada-Castro, M.M., Taboada-Castro, M.T., 2014. Hydrological response of a humid agroforestry catchment at different time scales. Hydrological Processes 28, 1677-1688. https://doi.org/10.1002/ hyp. 9714 
Rodríguez-Blanco, M.L., Taboada-Castro, M.M., Taboada-Castro, M.T., 2019. An overview of patterns and dynamics of suspended sediment transport in an agroforest headwater system in humid climate: results from a long-term monitoring. Science of Total Environment 648, 33-43. https://doi.org/10.1016/j.scitotenv.2018.08.118

Rodrigo, F.S., Trigo, M., 2007. Trends in daily rainfall in the Iberian Peninsula from 1951 to 2002. International Journal of Climatology 27, 513-529. https://doi. org/10.1002/joc. 1409

Salmi, T., Määttä, A, Anttila, P., RuohoAirola, T., Amnell, T., 2002. Detecting trends of annual values of atmospheric pollutants by the Mann-Kendall test and Sen's slope estimates - the excel template application MAKESENS. Publication on Air Quality No 31, Finnish Meteorological Institute, Air Quality Research, Helsinki, Finland.

Stahl, K., Hisdal, H., Hannaford, J., Tallakse, L.M., van Lannen H.A.J., Sauquet, E., Demuth, S., Fendekova, M., Jodar, J., 2010.Streamflow trends in Europe: evidence from a dataset of near-natural catchments. Hydrology
Earth System Science 14, 2367-2382. 0.5194/hess-14-2367-2010. https://doi. org/0.5194/hess-14-2367-2010

Svensson. C., Kundzewicz, W.Z., Maurer, T., 2005. Trend detection in river flow series: 2. Flood and low-flow index series. Hydrological Science Journal 500, 811-824. https://doi.org/10.1623/ hysj.2005.50.5.811.

Villarini, J.A., Smith, F., Serinaldi, A., Ntelekos, A., 2011. Analyses of seasonal and annual maximum daily discharge records for central Europe. Journal of Hydrology 399, 299-312. https://doi. org/10.1016/j.jhydrol.2011.01.007.

Wilson, D., Hisdal, H., Lawrence, D., 2010. Has streamflow changed in the Nordic countries? - Recent trends and comparisons to hydrological projections. Journal of Hydrology 394, 334-346. https://doi. org/10.1016/j.jhydrol.2010.09.010

Zabaleta, A., Morales, T., Meaurio, M., Gorria, C., Antigüedad, I., 2013. Regional hydrological signs for climate change in Southern Europe (Basque Country). Proceedings of the 5th Conference of Water, Climate and Environment, 1-11. BALWOIS 2012. Republic of Macedonia. 

